\title{
Tuberculous otitis Media, Petrous Apicitis, Brain Abscess And Meningoencephalitis in A 16-Month-Old Child
}

\author{
Popivanova $\mathrm{N}^{1}$, Baltadzhiev $\mathrm{I}^{1}$, Kazakova $\mathrm{Ts}^{2}$, Dineva $\mathrm{A}^{2}$ \\ ${ }^{I}$ Department of Infectious Diseases, Parasitology and Tropical Medicine, Faculty of Medicine, \\ Medical University, Plovdiv, Bulgaria; \\ ${ }^{2}$ St. George University Hospital, Plovdiv, Bulgaria
}

\begin{abstract}
Background: Tuberculous otitis media is very rare in the developed world and usually appears as a consequence of lung tuberculosis. Currently, Mycobacterium tuberculosis is the etiological agent in less than $0.1 \%$ of the cases of otitis media chronica.

Case presentation: We report a rare and fatal case of a child with tuberculous otitis media and petrous apicitis, extended to brain abscess and meningoencephalitis. The patient presented with meningeal signs, facial nerve palsy, bloody-purulent ear discharge, hearing loss, and abducens nerve palsy - a clinical picture of Gradenigo's syndrome. The cerebrospinal fluid cultured positive for M.tuberculosis. QuantiFERON-TB GOLD assay proved positive.

Conclusion: In the antibiotic era, tuberculosis of the middle ear with CNS involvement is a diagnostic challenge and clinicians should be aware of similar association not only in the developing countries, but especially in the industrialized world, since the disease is very rare and may result in delayed treatment with fatal consequences.

Key words: Tuberculosis, otitis media, petrous apicitis, brain abscess, meningoencephalitis
\end{abstract}

\section{Introduction}

Tuberculous otitis media (TOM) was described in 1883, only a year after the identification of Mycobacterium tuberculosis by $R$. Koch. A century later, only 3 to $5 \%$ of the cases of chronic otitis media were due to tuberculosis (TB) [1]. TOM is very rare in the developed world and is usually caused by bacterial associations or appears as a consequence of lung tuberculosis. Currently, M.tuberculosis is the etiological agent in less than $0.1 \%$ of the cases of otitis media chronica. In the pre-antibiotic era, the condition resulted in petrous apicitis, usually progressing to meningitis, brain abscess, or thrombosis of the cavernous sinus, and finally lethal outcome [2]. Currently, tuberculosis of the middle ear is a diagnostic challenge especially in the developed world, since the disease is very rare and may result in delayed treatment and extension of the infection in CNS with fatal consequences [3].

\section{Case presentation}

A 16-month-old Roma girl was referred to the University Clinics of Infectious Diseases from a District Hospital. She was diagnosed with middle ear infection and meningoencephalitis. The girl was the ninth child in a low income family of Gypsy ethnic minority, living in poor sanitation. The medical records indicated that she was born through a cesarean section with birth weight of 4 kilos. From the age of 5 months, the infant has suffered repeated pneumonia, later accompanied by purulent otitis media and has received antibiotic courses of ceftriaxone and amikacin. Upon admission, she weighted $3.1 \mathrm{~kg}$, was febrile, dyspneic and bradyarrythmic. Muco-purulent bloody discharge was noted from the right ear; left ear discharge was scarce, however, hearing seemed completely damaged. Several small cervical lymph nodes were palpable bilaterally. The child produced spontaneous cries and was convulsive upon pain stimulation. Meningeal irritation signs, right-sided facial palsy (House-Brackmann grade V) and right-sided abducens nerve palsy were present. Instrumental and microbiological analyzes: opacities.

The initial chest radiograph was interpreted by the radiologists as right-sided residual inflammatory

Head CT findings: Low density, likely inflammatory lesion sized $2 / 2.5 \mathrm{~cm}$, with low grade compression of the right anterior horn of the lateral ventricle.

Lumbar puncture (LP): Cerebrospinal fluid (CSF) changes are displayed in Table 1.

CT scan of temporal bones: Bilateral inflammation of the middle and inner ear; destruction of the front edge and apex of the right pyramid.

Retro-auricular trepanation of zona cribrosa: Bilateral extensive cavities, filled with caseous texture material, destructed bone and trabecular debris. On the right, the half destroyed incus was removed. The left incus was spared as little damage was present. The tympanic cavity was filled with caseous material and 
granulation tissue; tympanic membrane perforations were present in the posterior-superior quadrant. Dura mater exposure showed no pathological changes.

Microbiology: Nasal discharge was positive for methicillin resistant Staphylococcus aureus (sensitive to amikacin and clindamycin). Ear discharge grew Proteus (sensitive to ceftriaxone) and Enterococcus fecium (sensitive to vancomycin); Conventional CSF-cultures were negative for Gram's and acid fast bacilli (AFB).

Table 1 Dynamics of the cerebrospinal fluid changes of the child with tuberculous meningitis

\begin{tabular}{|c|c|c|c|c|c|c|c|}
\hline CSF & Normal & I week & II week & III week & IV week & V week & VI week \\
\hline WBC count & $\begin{array}{c}0-5 \\
\mathrm{x} 10^{6} / \mathrm{L}\end{array}$ & 57 & 18 & 267 & 186 & 136 & 34 \\
WBC & $\begin{array}{c}\text { No predomi- } \\
\text { nance }\end{array}$ & $\begin{array}{c}18 \% \mathrm{PMN} \\
82 \% \mathrm{Ly}\end{array}$ & N.A. & $\begin{array}{c}85 \% \mathrm{PMN} \\
15 \% \mathrm{Ly}\end{array}$ & $\begin{array}{c}18 \% \mathrm{PMN} \\
82 \% \mathrm{Ly}\end{array}$ & $\begin{array}{c}2 \% \mathrm{PMN} \\
98 \% \mathrm{Ly}\end{array}$ & $\begin{array}{c}22 \% \mathrm{PMN} \\
78 \% \mathrm{Ly}\end{array}$ \\
differential & $0.18-0.45 \mathrm{~g} / \mathrm{L}$ & 0.68 & 0.86 & 2.4 & 1.17 & 1.22 & 0.83 \\
Protein & $\begin{array}{c}2.5-3.5 \\
\mathrm{mmol} / \mathrm{L}\end{array}$ & 1.7 & 1 & 1.1 & 1.3 & 1.7 & 1.6 \\
\hline
\end{tabular}

Legend: CSF: cerebrospinal fluid; WBC: white blood cells; Ly: lymphocytes; N.A.: not available; $\mathrm{PMN}$ - polymorphonuclear cells

Treatment was initiated with broad spectrum antibiotic combination, based on sensitivity testing. The second LP showed a threefold decrease in the cell counts, however, the ear discharge persisted, the child's condition deteriorated and finally progressed to a coma. In order to verify the diagnosis repeated chest X-rays (Fig.1), and head CT were performed (Fig. 2), as well as a third LP (Table 1). Because the results worsened, a suspicion for tuberculous infection was raised. Then a treatment with streptomycin, isoniazid, rifampicin and ethambutol was initiated. The QuantiFERON-TB GOLD assay proved positive. The initial CSF-samples cultured positive for M.tuberculosis, but the growth process has been slow and the result was achieved six weeks post admission. The strain was sensitive to all four therapeutic agents. PCR testing, in terms of starting anti-TB therapy, has demonstrated negative for M.tuberculosis, but the experience of some authors showed that the treatment should not be ended even in case of negative results of PCR test [4]. Regardless of the appropriate anti-tuberculosis therapy and the significant reduction of pathologic changes in the final CFS sample, the child remained unconscious with decerebrate rigidity. Our endeavors failed to prevent the fatal outcome, which occurred 50 days after admission.

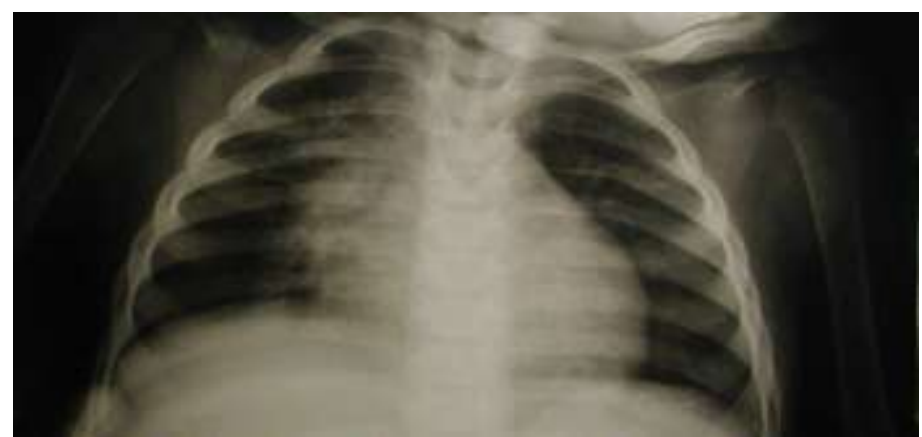

Figure 1. Chest X-rays: To the right, opacity adjacent to the heart is present, involving all pulmonary segments and extending to the hilar area.

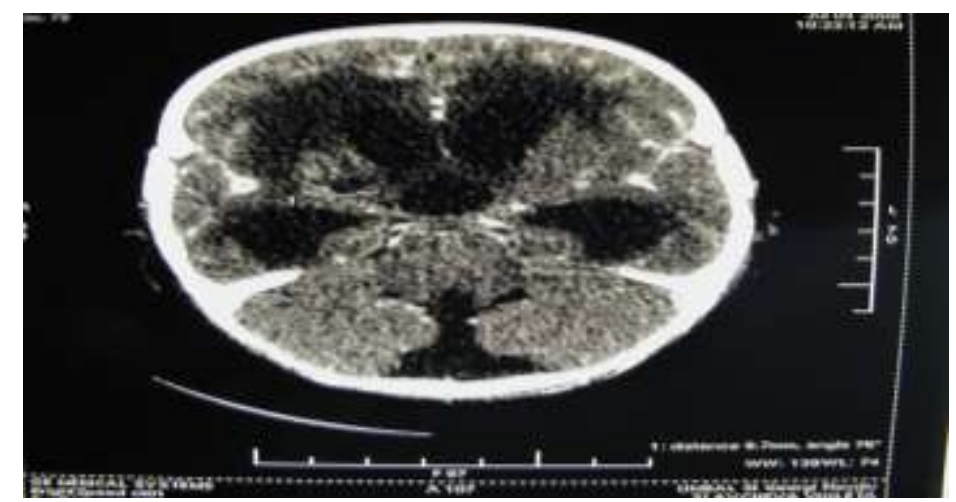


Figure 2. Contrast enhanced CT scan of the head: In the right brain hemisphere a destruction sized $12 / 15 \mathrm{~mm}$, surrounded by perifocal oedema and adjacent to the ventricles is present. Conclusion: Brain abscess in the right peri-ventricular zone, close to the anterior horn of the right lateral ventricle; progressive symmetrical internal hydrocephalus, involving the whole ventricular brain system.

\section{Final diagnosis}

Hematogenous disseminated pulmonary tuberculosis: bilateral subacute otitis media, otoantritis, and right-sided petrous apicitis, which progressed to ipsilateral brain abscess and tuberculous meningoencephalitis, complicated by internal hydrocephalus.

\section{Discussion}

The incidence rate of tuberculosis in Bulgaria is medium compared to the high incidence rates in Eastern European and Central Asian countries, and the low incidence rate in Western Europe. High risk groups include: Roma ethnic minority, individuals with alcohol and drug abuse, migrants and refugees, and individuals living with HIV/AIDS. Epidemiological data indicate that most newly diagnosed pediatric cases of the disease are attributable to contacts with adult patients with tuberculosis. Based on the National Immunization Schedule the initial BCG (Bacillus Calmette-Guérin) vaccination is mandatory and is performed 48 hours after delivery. Seven months old infants, who lack a visible BCG-vaccine scar, receive tuberculin-PPD (purified protein derivative) skin test and those who test negative are re-immunized. PPD negative individuals are given an additional boost at 7, 11 and 17 years of age [5]. In the past, the most common form of tuberculosis in children was the primary complex. Currently, tuberculous bronchadenitis is most frequently diagnosed, followed by the primary complex and latent tuberculosis; the remaining forms appear more rarely. In children, the diagnosis of tuberculosis requires at least two of the following criteria [6]: a history of contact with a proven TB case; hyperreactive tuberculin PPD skin test; specific changes on chest radiograph: primary complex, TB adenitis, miliary tuberculosis, and primary TB cavitation. Definite diagnosis is achieved based on positive for M.tuberculosis biological material from the patient. The introduction of rapid TB diagnostic tests is crucial for the timely start of anti-tuberculous treatment.

The BCG scar was not identified in our patient and the PPD skin test was negative - obviously the child had not been vaccinated or may be reacted anergic. His mother's chest radiograph was normal and she declined TB contacts in the family. The initial chest X-ray of the child failed to identify changes consistent with the above mentioned forms of tuberculosis.

The main symptom of TOM is painless ear discharge, not responding to the conventional antibiotic therapy. As infection progresses, multiple tympanic perforations develop, accompanied by abundant granulation tissue formation, bone necrosis and periauricular lymph node enlargement [1,3]. Similar symptoms and signs were also present in our patient. The misleading diagnosis in this case was related to the isolation of purulent bacteria from the ear and nasal discharges. For this reason, no TB microbiology cultures were performed and no AFB was sought in the ear discharge and in the biopsy material. However, the deteriorating condition of the child and the continued bloody-mucopurulent ear discharge not responding to conventional broad-spectrum antibiotics raised the suspicion of tuberculous infection. Yet there was a history of recurrent pneumonia (presumably tuberculosis), may be partially influenced due to the presence of amikacin in the therapeutic regimens.

Petrous apicitis is an intra-temporal complication of chronic otitis media. It involves the mucus surfaces of the temporal bone. The infection spreads via the diploic air spaces or runs along the venous plexuses. Tuberculosis is a very rare cause of petrous apicitis [7]. Most cases are attributable to purulent infection, and a few cases of tuberculous petrous apicitis have been described. The latter are mostly due to complicated pulmonary tuberculosis and are rarely primary [8]. The petrous apex is anatomically closely related to the eye branches of the trigeminal nerve and n.abducens. The latter innervates a single muscle, the lateral rectus bulbi. For this reason the classical consequences of petrositis are ear discharge, retro-bulbar pain and paralysis of $\mathrm{m}$. rectus lateralis bulbi. The triad of symptoms has been described as Gradenigo's syndrome [9]. The number of reported cases of tuberculous Gradenigo syndrome is scarce. We believe our patient presented one of these rare cases: the child's right eyeball was involuntary fixed in the midline as a consequence of ipsilateral abducens nerve palsy.

Peripheral facial nerve paralysis also belongs to the classic symptoms of petrositis and otoantritis [10]. Association with facial nerve paralysis has been described in $39 \%$ to $45 \%$ of the cases in children and should always be considered in patients with chronic ear discharge, especially in patients from certain ethnic minorities or immigrants, as tuberculosis is more common among these populations. Late complications of the infection include: labyrintitis, post-auricular fistulas, sub-periosteal abscesses and intra-cranial extension - brain abscesses and meningoencephalitis. The high risk of intra-cranial spread should not be neglected, considering the corresponding anatomic nervous and vascular structures. However, a few cases of meningoencephalitis 
associated with tuberculous otitis media and subsequent petrositis have been observed [11]. In Bulgaria we first reported the case on the V-th National Conference of Infectious Diseases [12, 13]. A frequent complication of TB meningo-encephalitis is the development of hydrocephalus, which carries a grim prognosis [14].

\section{Conclusion}

Even in the antibiotic era tuberculosis rare forms should not be neglected by infectious disease specialists, pediatricians and doctors in general practice. Tuberculosis of the middle ear with CNS involvement is a diagnostic challenge and clinicians should be aware of similar association not only in the developing countries, but especially in the industrialized world, since the disease is rare and may result in delayed treatment with fatal consequences. The early diagnosis requires a high index of clinical suspicion and specific therapy should initiate as early as possible to prevent complications and dissemination of infection.

\section{Acknowledgements}

We would like to thank prof. T. Shmilev for his advice regarding the combination of anti-tuberculosis medications of the child.

Conflicts of interest: All the authors declare that there are no financial or personal conflicts of interest.

\section{Reference}

[1]. Adhikari P. Tuberculous Otitis Media: A Review of Literature. The Internet Journal of Otorhinolaryngology. 2008;9(1):7 pages; ispub.com/IJORL/9/1/10058

[2]. Benedict RF, Cruz OLM, Morimoto And Ramas CC, Siebert Miniti DA. Tuberculosis of the temporal bone. Current state and presentation of 2 cases. Rev Bras Otolaryngol 1987;53:90-5.

[3]. Dale OT, Clarke AR, Drysdale AJ. Challenges encountered in the diagnosis of tuberculous otitis media: case report and literature review. J Laryngol Otol. 2011; 125(7):738-40.

[4]. Piekarska A, Kuydowicz J. [Diagnostic difficulties in tuberculosis meningoencephalitis]. Pneumonol Alergol Pol. 2002;70(910):504-8

[5]. European Centre for Disease Prevention and Control. Report of the joint ECDC and WHO review of the national tuberculosis programme in Bulgaria. Stockholm: ECDC; 2014. doi 10.2900/37158

[6]. Khan E, Starke JR. Diagnosis of tuberculosis in children: increased need for better methods. Emerg Infect Dis.1995;1(4):115-23.

[7]. Kearns DB, Coker NJ, Pitcock JK, Jenkins HA. Tuberculous petrous apicitis. Arch Otolaryngol. 1985;111(6):406-8.

[8]. Sethi A, Sabherwal A, Gulati A, Sareen D. Primary tuberculous petrositis. Acta Otolaryngol. 2005;125(11):1236-9.

[9]. Favier M, Bessou P, Franco-Vidal V, Pédespan JM. [Gradenigo syndrome and petrositis in a child]. Arch Pediatr. 2015;22(3): 2836.

[10]. Gupta N, Dass A, Goel N, Tiwari S. Tuberculous Otitis Media Leading to Sequential Bilateral Facial Nerve Paralysis. Iran J Otorhinolaryngol. 2015;27(80):231-7.

[11]. Mongkolrattanothai K, Oram R, Redleaf M, Bova J, Englund JA. Tuberculous otitis media with mastoiditis and central nervous system involvement. Pediatr Infect Dis J. 2003;22(5):453-6.

[12]. Popivanova N, Kazakova Ts, Dineva A, Baltadzhiev I. Tuberculous otitis media, petrous apicitis, and tuberculous meningoencephalitis. V-th National Conference of Infectious Diseases, October 1-3 2009, Hissar, Bulgaria.

[13]. Popivanova N, Kazakova Ts, Dineva A, Baltadzhiev I. Tuberculous otitis media, petrous apicitis, and tuberculous meningoencephalitis - literature review in the context of a case. In: Acute infections of the central nervous system, ed. MU-Plovdiv, 2010: 105-106.

[14]. Garlicki A, Kluba-Wojewoda U, Bociaga-Jasik M, Kalinowska-Nowak A, Sobczyk-Krupiarz I, Mach T. [Tuberculous meningoencephalitis--own observations]. Folia Med Cracov. 2003;44(1-2):27-38. 